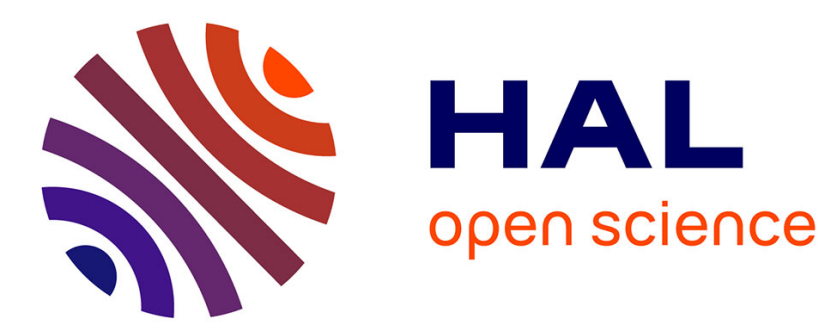

\title{
A multi-objective Gray Wolf algorithm for routing in IoT Collection Networks with real experiments
}

\author{
Sihem Tlili, Sami Mnasri, Thierry Val
}

\section{To cite this version:}

Sihem Tlili, Sami Mnasri, Thierry Val. A multi-objective Gray Wolf algorithm for routing in IoT Collection Networks with real experiments. Netherlands' Catalysis and Chemistry Conference (NCCC 2021), Taif University; IEEE Western Saudi Arabia Section; IEEE Western Saudi Arabia Section Computer Society Chapter, Mar 2021, Taif, Saudi Arabia. hal-03169459

\section{HAL Id: hal-03169459 \\ https://hal.science/hal-03169459}

Submitted on 15 Mar 2021

HAL is a multi-disciplinary open access archive for the deposit and dissemination of scientific research documents, whether they are published or not. The documents may come from teaching and research institutions in France or abroad, or from public or private research centers.
L'archive ouverte pluridisciplinaire HAL, est destinée au dépôt et à la diffusion de documents scientifiques de niveau recherche, publiés ou non, émanant des établissements d'enseignement et de recherche français ou étrangers, des laboratoires publics ou privés. 


\section{A multi-objective Gray Wolf algorithm for routing in IoT Collection Networks with real experiments}

\author{
Sihem Tlili \\ UT2J, CNRS-IRIT (RMESS), University \\ of Toulouse, \\ Toulouse, France \\ Dept. of Computer Sciences, Faculty of \\ Science of Gafsa, University of Gafsa, \\ Gafsa, Tunisia \\ sihem.tlili@gmail.com
}

\author{
Sami Mnasri \\ CNRS-IRIT (RMESS), University of \\ Toulouse, \\ Toulouse, France \\ Sami.Mnasri@irit.fr \\ Dept. of Computer Sciences, \\ Community College, University of \\ Tabuk, Tabuk, KSA \\ smnasri@ut.edu.sa
}

\author{
Thierry Val \\ UT2J, CNRS-IRIT (RMESS), University \\ of Toulouse, \\ Toulouse, France \\ val@irit.fr
}

\begin{abstract}
The routing is a real-world engineering problem in IoT Collection Networks. It has a direct impact on the network performance. The routing problem can be treated as a multi-objective optimization problem. Our objective is then to study the IoT routing problem using the Multi-Objective Grey Wolf Optimizer (MOGWO) [1], which consists in translating the routing process into a multi-objective optimization problem and implementing its execution with the MOGWO. In this context, the experimental study showed that the MOGWO provides better quality of routing (higher lifetime of the network, more efficient delivery delay and higher number of neighbors).
\end{abstract}

Keywords-IoT routing, IoT Collection Networks, Multiobjective Grey Wolf, Prototyping, Optimization

\section{INTRODUCTION}

Nowadays, several application areas are affected by the Internet of Things (IoT). Indeed, they take advantage of its benefits, such as efficiency, greater control, security, etc. In fact, the components of the IoT network are organized according to a topology the choice of which is a very important factor among those related to the well-functioning and efficiency of the network. Actually, several topologies are possible. The most common topologies are: point to point topology (Fig. 1(a)), mesh topology (Fig. 1(b)), star

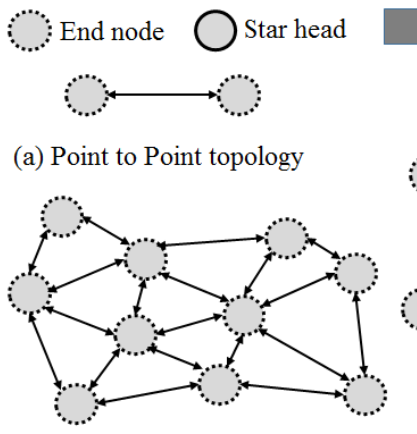

(b) Mesh topology

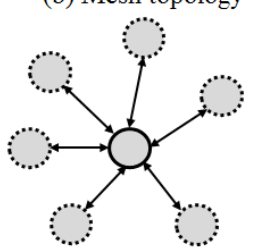

(c) Star topology

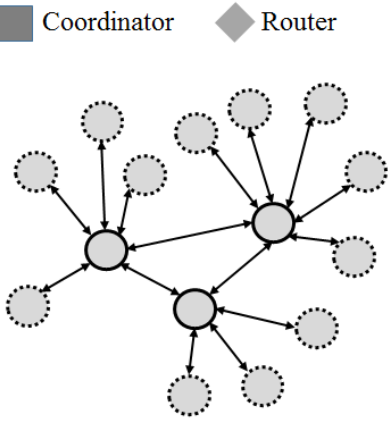

(d) Hybrid topology

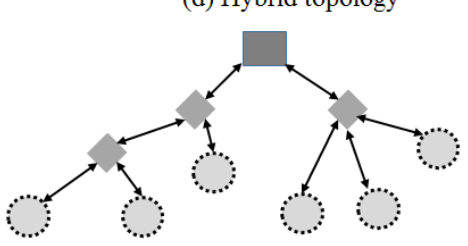

(e) Tree topology

Fig. 1. The most common topologies for IoT networks topology (Fig. 1(c)), hybrid topology (Fig. 1(d)) and tree topology (Fig. 1(e)).

In fact, the topology used to organize the network components is among the important factors that serve to specify the routing strategy to be applied. The transfer of data between the source and the destination takes place along a selected path. The choice of the appropriate path is made by applying a routing protocol the efficiency of which has a big effect on the growth of the IoT [2]. Indeed, several network performance criteria, such as data rate, loss rate, network lifetime, delivery delay, etc., are influenced by the used routing protocol. In real applications, such as smart city, transport, industry, etc., since the IoT environments are more and more complex, the task of routing becomes more and more difficult.

Indeed, several challenges are related to the definition of the routing strategy the most important of which are the limited capacities of the objects, the heterogeneity of the network components, security, failure management, transfer reliability, support for dynamic topologies, etc. Therefore, to evaluate the performance of the applied routing protocol, several metrics including the consumed energy, the total path delay, the loss rate, etc., are defined. The definition of these evaluation metrics and the constraints to be satisfied makes it possible to translate the routing task into an optimization problem. The path to be used for the routing of the data from the source to the destination is defined through the resolution of the built optimization problem. In fact, the construction of an optimization problem consists in defining a number of decision variables, their value domains, a set of constraints and a number of objective functions. The possible values of the variables constitute a search space. The resolution of the problem serves to seek an optimal solution in this space. This solution must optimize the objective functions and satisfy the defined constraints. Besides, optimization brings better performance and success in several areas of the real world. It is therefore useful to improve the routing performance in IoT environments based on optimization. In other words, the routing task can be modeled and solved as an optimization problem to take advantage of this paradigm success.

As a matter of fact, the objective of this paper is to demonstrate the ability of optimization to remedy the complexity of routing and therefore improve the performance of IoT networks. For this reason, the Multi-Objective Gray Wolf Optimizer (MOGWO) [1] is used to solve routing in an IoT environment. 
The remainder of this paper contains three sections. In section 2, we present recent works that propose routing strategies in IoT environments based on optimization algorithms. Section 3 describes our proposal to use the MOGWO algorithm to solve the routing problem. In section 4 , the performance of the proposed algorithm is tested and compared to the Nondominated Sorting Genetic Algorithm III (NSGA-III) [3].

\section{RELATED WORKS}

There are some research studies that used optimization algorithms to solve the routing problem in the IoT networks.

In fact, the Multi-objective Imperialist Competitive Algorithm (MOICA) proposed in [4] is based on the Imperialist Competitive Optimization Algorithm (ICOA) [5]. The routing task is modeled as an optimization problem constructed with the following parameters: integrity, confidentiality and availability, while the MOICA is a secure routing protocol the main objective of which is to reduce the probability of piracy. The contribution of the used optimization algorithm is reflected in the results of the carriedout tests although these tests are carried out in a small mesh network (20 nodes). In addition, the presented results do not contain comparisons with other protocols.

On the other hand, the IoT-MANET networks contain IoT components that are connected to Mobile Ad-hoc Network (MANET). In [6], the service quality of these networks is improved based on the Intelligent Water Drop Optimization Algorithm [7] and the Firefly Optimization Algorithm [8]. Moreover, the conducted simulations gave results that proved the contribution of these two algorithms. The evaluated metrics are the data rate, the transmission delay, the percentage of successfully transmitted packets. The obtained results showed the improvement of these metrics. On the other hand, this proposal does not address the energy consumption, which is a very important challenge for the IoT. Besides, it does not support i) the self-stabilization (act automatically in case of problems) and ii) the identification of malicious nodes that are capable of disrupting the routing. These two challenges, which are cited in [9], are considered as the most important ones in the IoT-MANET networks.

For their part, the authors in [10] used the Shuffled Frog Leaping Optimization Algorithm (SFLA) to solve the routing of multimedia data in an IoT network. This algorithm consists of content-based, multi-hop routing. As for the simulations, they were carried out to evaluate the data rate, the consumed energy and the percentage of successfully transmitted packets. Although the proposal does not support the data redundancy, this challenge has a great effect on the performance of a multimedia network [11] since data redundancy can consume the resources available in the network.

On the other hand, the Heterogeneous Modified Grey Wolf Optimizer (HMGWO) [12] is a routing protocol that uses the Gray Wolf Optimizer (GWO) [13]. HMGWO is applied in a network composed of fixed and heterogeneous nodes since they have different energy capacities. Then, the carried out simulations showed improvements in terms of data rate and consumed energy. Although the optimizing energy consumption improves the lifetime of network components, its performance is not validated by real experiments. On the other hand, energy consumption is the only parameter taken into consideration by the HMGWO [12]. In addition, this protocol does not support the management of mobile entities.
Furthermore, the HMGWO algorithm [12] is not interested in data exchanges between communicating objects and between star heads. The studied environment contains only communication between a base station and the star heads.

\section{Routing RESOLUTION WITH THE MUlti-OBJECTIVE GREY WOLF OPTIMIZER ALGORITHM}

\section{A. The Multi-Objective Grey Wolf Optimizer algorithm}

The authors of [13] propose an optimization algorithm called the Gray Wolf Optimizer (GWO). In fact, the proposed algorithm is inspired by the behavior of gray wolves when hunting. Therefore, in the GWO algorithm, the optimization problem is the hunting process, where:

- The optimum of the problem is the prey.

- $\quad\{X i\}(i=1,2, \ldots, n)$ is a search space, which consists of the wolves that represent the search agents of the problem. Each wolf is represented by its coordinates in the studied environment (a vector $\vec{X} \imath$ ).

- $\quad$ the optimal solutions are wolves called $\alpha, \beta$ and $\delta$.

- the best solution is the $\alpha$ wolf.

- $\quad$ the $\omega$ wolves are the other search agents.

- $\omega$ wolves are dominated by $\alpha, \beta$ and $\delta$ wolves that guide the hunt. This dominance comes from the fact that $\alpha, \beta$ and $\delta$ wolves have a better knowledge of the prey's position. Indeed, the wolves move in order to encircle it where $\omega$ wolves follow $\alpha, \beta$ and $\delta$ wolves by moving in order to get closer to the prey. Moving a wolf is changing its coordinates. Its new position is then calculated based on its previous position. This calculation is done using three vectors $\vec{a}, \vec{A}$ and $\vec{C}$. The GWO algorithm presents the method used to choose and calculate the components of these vectors. In fact, the purpose of this method is to get closer and closer to the prey.

MOGWO approach [1] is a multi-objective version of the GWO algorithm [13], which adds two concepts:

- Non-dominated solutions are stored in an archive.

- A comparison of the solutions, which is performed according to a proposed mechanism, is used to select the optimal solutions $X_{\alpha}, X_{\beta}$ and $X_{\delta}$ for the purpose of orienting the research towards unexplored research agents.

\section{B. The Multi-Objective Grey Wolf Optimizer for the Internet of Things routing}

1) The network model: In this proposal, we consider a network with an organization carried out according to a hybrid topology and divided into stars, each of which has a head. The star heads communicate with one another according to a mesh organization. In other words, if an object wants to send a message, the routing begins by sending the message to the head of the star to which it belongs then, the message is passed from one head to another using a multi-hop routing. Then, if the destination does not belong to the IoT network, multi-hop routing ends when the message reaches a gateway to the outside, otherwise it ends when it arrives at the star that contains the final destination. The possible 
communication in this network model is exchanged between objects and data processing centers, inter-objects and with an external destination.

2) Routing resolution by the Multi-Objective Grey Wolf Optimizer algorithm: Suppose that :

- $\quad S_{1}$ and $S_{2}$ are two stars in the studied network.

- $\quad H_{1}$ and $H_{2}$ are the heads of $S_{l}$ and $S_{2}$, respectively.

- $O_{1}$ and $O_{2}$ are two communicating objects, $O_{1} \in S_{I}$ and $\mathrm{O}_{2} \in \mathrm{S}_{2}$.

- $B$ is a gateway to the outside.

- $O_{1}$ wants to send a message $m$ to $O_{2}$ (or to $B$ if the final destination is not in the network).

Therefore, we propose to solve the routing with the MOGWO algorithm where the routing task is the optimization problem and the optimum of the problem is the destination. The objective functions must take into consideration the routing challenges while the search agents are the heads of the stars. Indeed, the routing to be carried out is multi-hops between the star heads where each iteration of the algorithm selects the destination of the next hop. Therefore, the proposed routing resolution is presented as follows:

- The message $\mathrm{m}$ is sent from $O_{1}$ to $H_{l}$

- If $O_{2} \in S_{1}, m$ is sent from $H_{1}$ to $O_{2}$.

- Otherwise, multi-hop routing will be applied between the heads of the stars. $H_{1}$ is the $X_{\text {current }}$, which determines the head to reach in the first hop (the next $\left.X_{\text {current }}\right)$.

a. Search agents are initialized by neighboring star heads to construct $\left\{X_{i}\right\}$.

b. The values of the objective functions are calculated for each $X_{i}$.

c. The elements of $\left\{X_{i}\right\}$, which represent nondominated solutions, are determined to initialize an archive.

d. The $X_{\alpha}, X_{\beta}$ and $X_{\delta}$ which represent the best solutions in the archive are selected.

e. The message $m$ is sent to $X_{\alpha} . X_{\alpha}$ also receives the coordinates of $X_{\beta}, X_{\delta}$ and the elements of $\left\{X_{i}\right\}$.

f. $X_{\alpha}$ is therefore the $X_{\text {current }}$.

g. While $\left(X_{\text {current }} \neq H_{2}\right.$ (or $\left.\left.X_{\text {current }} \neq B\right)\right)$ do :

i. The coordinates of the elements of $\left\{X_{i}\right\}$ are changed using the coordinates of the three best solutions $X_{\alpha}, X_{\beta}$ and $X_{\delta}$. MOGWO provides the equations for these changes.

ii. For each element of $\left\{X_{i}\right\}$ : replace its coordinates with those of the nearest star head.

iii. For each element of $\left\{X_{i}\right\}$ : calculate its objective functions' values.

iv. Determine the elements of $\left\{X_{i}\right\}$ that represent non-dominated solutions.

v. The archive is updated with the determined non-dominated solutions. vi. The new solutions $X_{\alpha}, X_{\beta}$ and $X_{\delta}$ are selected, the MOGWO algorithm provides the method to search for these solutions in the archive.

$X_{\alpha}$ is the destination of the next hop.

vii. The message $m, X_{\beta}, X_{\delta}$ and the elements of $\left\{X_{i}\right\}$ are sent to $X_{\alpha}$.

viii. $\mathrm{X} \alpha$ is the $X_{\text {current }}$.

h. The last found $X_{\alpha}$ represents $H_{2}$ or $B$. The message $m$ is sent from $\mathrm{H}_{2}$ to $\mathrm{O}_{2}$, or to the outside by $B$.

\section{NUMERICAL AND EXPERIMENTAL EVALUATION}

In this section, the performance of the introduced MOGWO algorithm is assessed by evaluating its Hypervolume values compared to a recent multi-objective algorithm, the NSGA-III [3]. Then, the MOGWO performance is evaluated by prototyping tests on a real testber 7 ible 1 shows the used parameters for NSGA-III and MOGWU. Table II illustrates the parameters of the experiments.

Since the IoT routing is real-world engineering problem, the Hypervolume (HV) is used rather than the IGD to evaluate the behaviors of the optimizers [14]. Table III presents the best, average and worst values of the Hypervolume on 30 execution of the MOGWO and the NSGA-III for different number of iterations and objectives. Bolded values in Table III are the best (highest) HV values in each line.

TABLE I. SETTINGS OF NSGA-III AND MOGWO

\begin{tabular}{|c|c|c|c|}
\hline \multicolumn{3}{|c|}{ Parameter } & Value \\
\hline \multicolumn{3}{|c|}{ Size of population [Nbr of grey wolfs] } & 300 \\
\hline \multicolumn{3}{|c|}{ Number of runs } & $\begin{array}{l}\text { 30, using distinct } \\
\text { initial populations }\end{array}$ \\
\hline \multicolumn{3}{|c|}{ Number of objectives } & 3 to 5 \\
\hline \multicolumn{3}{|c|}{ Number of generations } & 10 to 650 \\
\hline \multirow{4}{*}{ MOGWO } & \multicolumn{2}{|c|}{ Archive size } & 50 \\
\hline & \multicolumn{2}{|c|}{ alpha } & 0.1 \\
\hline & \multicolumn{2}{|l|}{ beta } & 4 \\
\hline & \multicolumn{2}{|l|}{ gamma } & 2 \\
\hline \multirow{6}{*}{ NSGA-III } & \multirow[t]{3}{*}{ Mutation } & Operator & Swap Mutation \\
\hline & & distribution index & 45 \\
\hline & & probability & 0.0025 \\
\hline & \multirow[t]{3}{*}{ Cross-over } & Operator & $\begin{array}{l}\text { sequential } \\
\text { constructive (SCX) }\end{array}$ \\
\hline & & distribution index & 25 \\
\hline & & probability & 0.9 \\
\hline
\end{tabular}

TABLE II. PARAMETERS OF THE PROTOTYPING EXPERIMENTS

\begin{tabular}{|c|c|}
\hline Nodes number $=$ & $\begin{array}{l}22 \text { stationary nodes, } 1 \\
\text { mobile node }\end{array}$ \\
\hline Nodes repartition & $300 * 300 \mathrm{~m}^{2}$ \\
\hline $\begin{array}{c}\text { Received Signal Strength Indicator } \\
\text { (RSSI) }\end{array}$ & $\begin{array}{l}\text { Variab }^{1 \wedge \text { (initiated to } 120} \\
\mathrm{dBm})=\end{array}$ \\
\hline Frame Error Rate(FER) & $\begin{array}{l}\text { Variab initiated to } \\
0.01)=\end{array}$ \\
\hline Average number of runs & 25 \\
\hline Transmission range & $27 \mathrm{~m}=$ \\
\hline Sensing range & $27 \mathrm{~m}$ \\
\hline Transmission range & $27 \mathrm{~m}=$ \\
\hline Transmission power & $110 \mathrm{~mW}$ \\
\hline
\end{tabular}


TABLE III. HYPERVOLUME VALUES (BEST, AVERAGE AND WORST) FOR MOGWO AND NSGA-III ON THE ROUTING PROBLEM

\begin{tabular}{|l|l|l|l|}
\hline $\begin{array}{c}\text { Nbr of } \\
\text { objectives }\end{array}$ & $\begin{array}{c}\text { Nbr of } \\
\text { generations }\end{array}$ & NSGA-III & MOGWO \\
\hline \multirow{3}{*}{1} & 350 & 0.921185 & $\mathbf{0 . 9 2 1 5 9 4}$ \\
& & $\mathbf{0 . 9 1 6 8 7 4}$ & 0.914165 \\
2 & \multirow{3}{*}{450} & 0.881268 & $\mathbf{0 . 9 0 1 1 6 8}$ \\
\hline \multirow{3}{*}{3} & & $\mathbf{0 . 9 8 1 0 2 3}$ & 0.980005 \\
& 650 & 0.978096 & $\mathbf{0 . 9 7 9 0 8 7}$ \\
\hline & & 0.972858 & $\mathbf{0 . 9 7 3 2 6 7}$ \\
& & 0.972434 & $\mathbf{0 . 9 7 2 6 0 1}$ \\
\hline
\end{tabular}

Table III highlights the influence of the number of considered objectives on the quality of the solution. Indeed, when the number of objectives increases, the values of the Hypervolume decreases (becomes worst). Regarding the number of generations, higher Hypervolume values (better results) are obtained for higher number of generations. It is also noticed that MOGWO outperforms NSGA-III in terms of Hypervolume values in most cases.

Fig. 2 compares the behavior of the introduced MOGWO with the NSGA-III according to a set of network metrics indicating the quality of the proposed routing. The number of neighbors in the routing table is a metric indicating the quality of routing. The lifetime can be computed by the time in which the first IoT object shuts off. The lifetime is also an indicator showing the energy efficiency of the routing protocol. The delivery time indicate the time in which the transmitted message reaches its destination. The routing protocol and the number of hops considerably influences the delivery time. The RSSI represents the quality of the emitte $\downarrow S$ gnal. RSSI is better when the number of hops proposeu uy the routing protocol is less.

It can be deduced from Fig. 2(a) that MOGWO outperforms NSGA-III in terms of average number of neighbors which indicated a better quality of routing with MOGWO. When the number of iterations exceeds 400 , the improvement of the number of neighbors becomes little for both algorithms which indicate that 400 iteration is the convergence value, especially for MOGWO.

Fig. 2(b) highlights the better performance of MOGWO compared with NSGA-III regarding the overall average lifetime of the network. This indicates that routing with MOGWO is more energy efficient than NSGA-III.

Fig. 2(c) $\overline{c o}$ nputes the average delivery time of the network messages. The NSGA-III seems to be better than NSGA-III regarding the needed ave ag: delivery time, especially for a high number of iwations. Here the convergence of the algorithms regarding the metric of delivery time is not reached even after 650 iterations.

Fig. 2(d) represents the average values of RSSI obtained after the routing using the NSGA-III and the MOGWO. The performance of the two algorithms considering the RSSI is comparable.

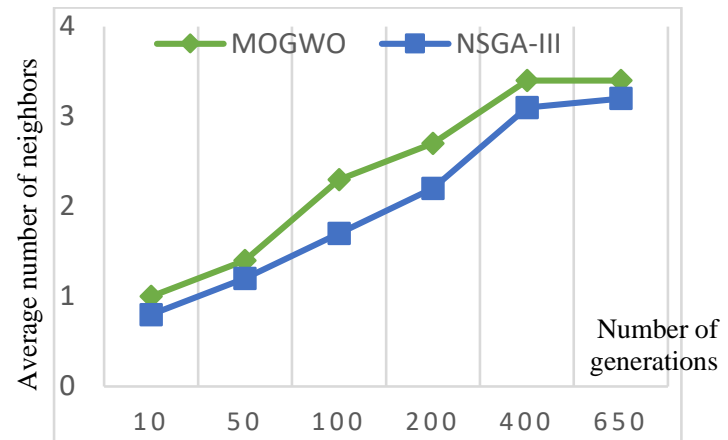

(a) Nbr.of neighbors' values of routing with MOGWO and NSGA-III

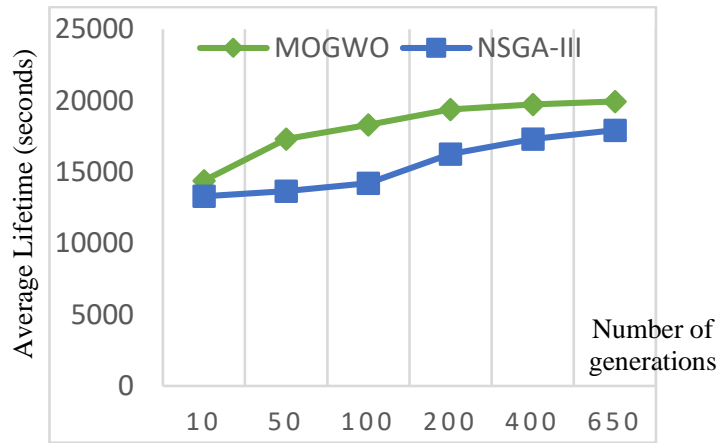

(b) Lifetime values of routing with MOGWO and NSGA-III

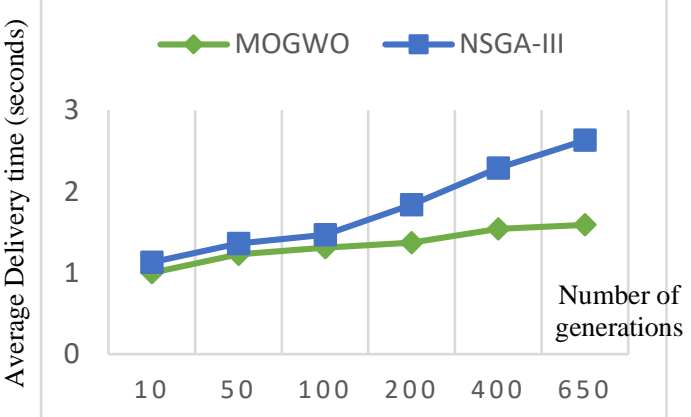

(c) Delivery time values of routing with MOGWO and NSGA-III

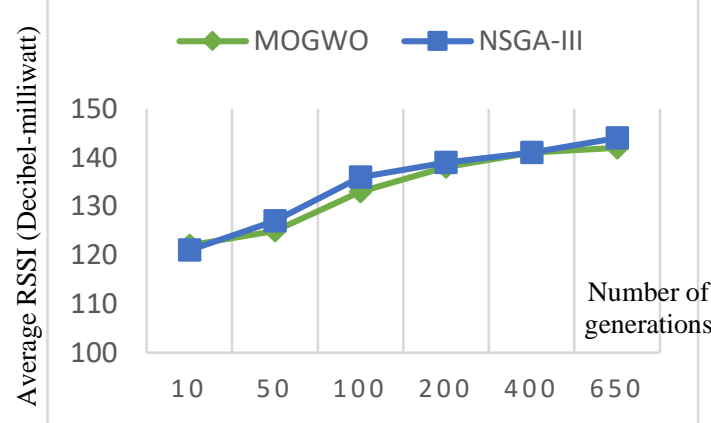

(d) RSSI values of routing with MOGWO and NSGA-III

Fig. 2. Network metrics values for routing with MOGWO and NSGA-III

\section{CONCLUSIONS}

In conclusion, we can say that this work is intended to demonstrate the ability of the optimization algorithms to overcome the routing challenges in IoT Collection Networks. In fact, this research has two objectives, the first consists in showing the efficiency of the existing routing strategies that are based on optimization. Indeed, these proposed protocols took advantage of the used optimization algorithms to improve the performance of the routing however, they are not efficient enough to meet the needs of the IoT routing.

The second objective is intended to propose a routing resolution in an IoT environment based on the Multi- 
Objective Grey Wolf Optimizer. In fact, we took advantage of the GWO algorithm, which has proven its effectiveness in large combinatorial problems to develop the proposed routing algorithm. We also carried out real experiments to compare the proposed routing algorithm to a recent multi-objective algorithm, the NSGA-III. The experimental results have shown that our proposal is more efficient than NSGA-III. The MOGWO algorithm outperforms it in terms of Hypervolume. In addition, the proposed routing algorithm is better than NSGA-III in terms of lifetime of the network (energy consumption), delivery delay and number of neighbors).

As continuation of this work, we are thinking of extending the objective functions of the constructed optimization problem in order to take into consideration more challenges for the IoT routing.

\section{REFERENCES}

[1] Seyedali Mirjalili, Shahrzad Saremi, Seyed Mohammad Mirjalili, and Leandro dos S. Coelho. Multi-objective grey wolf optimizer: A novel algorithm for multi-criterion optimization. Expert Systems with Applications, 47:106 - 119, 2016

[2] Shailja Agnihotri and K. Ramkumar. A survey and comparative analysis of the various routing protocols of internet of things. International Journal of Pervasive Computing and Communications, 13:00-00, 082017.

[3] K. Deb and H. Jain, "An Evolutionary Many-Objective Optimization Algorithm Using Reference-Point-Based Nondominated Sorting Approach, Part I: Solving Problems With Box Constraints," in IEEE Transactions on Evolutionary Computation, vol. 18, no. 4, pp. 577-601, Aug. 2014, doi: 10.1109/TEVC.2013.2281535.

[4] Alireza Ebrahimi Basabi, Jingsha He, and Seyed Mahmood Hashemi. Secure routing in iot with evolutionary algorithm. Journal of Advances in Computer Networks, 7(2), 2019.
[5] E. Atashpaz-Gargari and C. Lucas. Imperialist competitive algorithm: An algorithm for optimization inspired by imperialistic competition. In 2007 IEEE Congress on Evolutionary Computation, pages 4661-4667, 2007.

[6] Neha Sharma, Usha Batra, and Sherin Zafar. Remit accretion in iot networks encircling ingenious firefly algorithm correlating water drop algorithm. Procedia Computer Science, 167:551 - 561, 2020. International Conference on Computational Intelligence and Data Science.

[7] Basem Alijla, Li-Pei Wong, Chee Lim, Ahamad Tajudin Khader, and Mohammed Al-Betar. A modified intelligent water drops algorithm and its application to optimization problems. Expert Systems with Applications, 41:6555-6569, 112014.

[8] Xin-She Yang. Firefly algorithms for multimodal optimization. In Osamu Watanabe and Thomas Zeugmann, editors, Stochastic Algorithms: Foundations and Applications, pages 169-178, Berlin, Heidelberg, 2009. Springer Berlin Heidelberg.

[9] Jonny Karlsson, Laurence Dooley, and Goran Pulkkis. Secure routing " for manet connected internet of things systems. pages 114-119, 08 2018.

[10] Saeid Jedari Jazebi and A. Ghaffari. Risa: routing scheme for internet of things using shuffled frog leaping optimization algorithm. Journal of Ambient Intelligence and Humanized Computing, 11:4273-4283, 2020.

[11] Hang Shen and Guangwei Bai. Routing in wireless multimedia sensor networks: A survey and challenges ahead. Journal of Network and Computer Applications, 71:30 - 49, 2016.

[12] Xiaoqiang Zhao, Shaoya Ren, Heng Quan, and Qiang Gao. Routing protocol for heterogeneous wireless sensor networks based on a modified grey wolf optimizer. Sensors, 20:820, 022020.

[13] Seyedali Mirjalili, Seyed Mirjalili, and Andrew Lewis. Grey wolf optimizer. Advances in Engineering Software, 69:46 - 61, 032014.

[14] S. Mnasri, N. Nasri, A. van den Bossche, T. Val, A new multi-agent particle swarm algorithm based on birds accents for the 3D indoor deployment problem. ISA Transactions, 91:262-280, 2019, doi:10.1016/j.isatra.2019.01.026 